\title{
Neural and Glial Progenitor Transplantation as a Neuroprotective Strategy for Amyotrophic Lateral Sclerosis (ALS)
}

\author{
Amanda M. Haidet-Phillips ${ }^{1}$ and Nicholas J. Maragakis ${ }^{1 *}$.
}

${ }^{1}$ Department of Neurology, Johns Hopkins University, 250.10 Rangos Building, 855 North

Wolfe St., Baltimore, Maryland 21205.

*Corresponding author

Nicholas J. Maragakis, M.D.

Department Neurology

Johns Hopkins University, School of Medicine

855 N. Wolfe St., Rm 248

Baltimore, MD 21205

Tel: 410-614-9874

Fax: 410-502-5459

Email:nmaragak@jhmi.edu 


\section{$\underline{\text { Abstract }}$}

ALS is a neurodegenerative disease with a prevalence rate of up to $7.4 / 100,000$ and the overall risk of developing ALS over a lifetime is 1:400. Most patients die from respiratory failure following a course of progressive weakness. To date, only one traditional pharmaceutical agent - riluzole, has been shown to afford a benefit on survival but numerous pharmaceutical interventions have been studied in preclinical models of ALS without subsequent translation to patient efficacy. Despite the relative selectivity of motor neuron cell death, animal and tissue culture models of familial ALS suggest that non-neuronal cells significantly contribute to neuronal dysfunction and death. Early efforts to transplant stem cells had focused on motor neuron replacement. More practically for this aggressive neurodegenerative disease, recent studies, preclinical efforts, and early clinical trials have focused on the transplantation of neural stem cells, mesenchymal stem cells, or glial progenitors. Using transgenic mouse or rat models of ALS, a number of studies have shown neuroprotection through a variety of different mechanisms that have included neurotrophic factor secretion, glutamate transporter regulation, and modulation of neuroinflammation, among others. However, given that cell replacement could involve a number of biologically relevant factors, identifying the key pathway(s) that may contribute to neuroprotection remains a challenge. Nevertheless, given the abundant data supporting the interplay between non-neuronal cell types and motor neuron disease propagation, the replacement of disease-carrying host cells by normal cells may be sufficient to confer neuroprotection. Key preclinical issues that currently are being addressed include the most appropriate methods and routes for delivery of cells to disease-relevant regions of the neuraxis, cell survival and migration, and tracking the cells following transplantation. Central to the initial development of stem cell transplantation into patients with ALS is the demonstration that 
transplanted cells lack tumorigenicity and have the appropriate biodistribution to ensure the safety of ALS patients receiving these therapies.

Here, we review preclinical and clinical studies focusing on the transplantation of neural and glial progenitor cells as a promising neuroprotective therapy for ALS. The rationale for stem cell transplantation for neuroprotection, proof-of-concept animal studies, and current challenges facing translation of these therapies to the clinic is presented. Lastly, we discuss advancements on the horizon including induced pluripotent stem cell technology and developments for cellular tracking and detection post-transplantation. With the safe completion of the first-in-human Phase I clinical trial for intraspinal stem cell transplantation for ALS in the United States, the time is ripe for stem cell therapies to be translated to the clinic and excitingly, evaluated for neuroprotection for ALS. 


\section{$\underline{\text { Introduction }}$}

The unique abilities of stem cells to proliferate, regenerate, and renew has pushed them to the forefront of modern medicine. Stem cells are defined as cells that are capable of continuously dividing and as well as differentiating into multiple cell types. Stem cells range in diversity from embryonic stem cells which have the ability to become all three developmental germ layers to lineage-restricted progenitors which can only differentiate toward one cellular lineage. Due to their ability to regenerate cells of the central nervous system such as neurons, astrocytes, and oligodendrocytes, stem cells have been investigated for their therapeutic potential for neurological disease.

Amyotrophic Lateral Sclerosis (ALS) is a progressive, fatal, neurodegenerative disease caused by the death of upper and lower motor neuron pools in the cortex, brain stem, and spinal cord. ALS patients experience muscle atrophy and weakness due to lower motor neuron loss as well as spasticity and hypereflexia as a result of the loss of motor neurons in the motor cortex. Patients typically progress rapidly with the average length from disease onset to death ranging from 2-5 years following a diagnosis. The vast majority of ALS cases are sporadic. However, $10 \%$ of patients have a family history of disease (familial ALS). The indistinguishable nature of sporadic and familial forms of ALS gives hope that a single therapeutic strategy may be successful to treat both forms of the disease. Presently, there are no effective therapies with riluzole being the only FDA-approved drug for ALS which meagerly extends lifespan by several months in some patients (Bensimon $\mathrm{G}, 1994$ ). Due to the rapid progression and lethal nature of the disease, all avenues are being explored with the goal of developing a suitable therapeutic for ALS. One of the strategies currently in clinical development is stem cell transplantation to halt or slow ALS disease progression. 
The concept of stem cell therapy for ALS has long been considered and there is a rich literature focusing on the generation of motor neurons from stem cells (Nizzardo et al., 2010; Peljto and Wichterle, 2011). Since motor neuron loss is the prominent clinical and pathological feature in ALS, the original goal for stem cell transplantation was to replace dying motor neurons with healthy, stem-cell derived motor neurons. Indeed, motor neurons can be generated from neural and embryonic stem cell sources and transplanted to the spinal cord of a mouse model of familial ALS (Corti et al., 2007; Lopez-Gonzalez et al., 2009; Martin and Liu, 2007). However, multiple challenges exist in motor neuron replacement for humans such as replacing vulnerable neurons across the entire spinal cord and motor cortex, promoting rapid growth of motor axons out from the spinal cord, and establishing functional connections with muscles in the periphery.

Additionally, an even larger hurdle to overcome for motor neuron replacement may be the toxic environment of the ALS spinal cord. Although motor neurons die in ALS, a substantial number of studies now show that non-neuronal cell types become dysfunctional during disease and can directly initiate the death of otherwise healthy motor neurons. The majority of these studies derive from research using rodent models of ALS where mice ubiquitously overexpress a mutated version of the superoxide dismutase 1 (SOD1) gene linked to a familial form of ALS (Gurney et al., 1994). These mice develop motor neuron disease which closely mimics human ALS including motor neuron loss, muscle wasting, paralysis, and death. While these mice express the mutant SOD1 gene in all cell types, it was shown that expression of the mutant SOD1 only in neurons did not lead to the development of typical ALS, suggesting mutant SOD1 gene expression within non-neuronal cells also plays an active role in motor neuron disease in these mice (Jaarsma et al., 2008; Lino et al., 2002; Pramatarova et al., 2001). 
Subsequent studies have capitalized on cell-type restricted promoters to induce specific excision of the mutant SOD1 gene within certain cellular lineages. Removal of the mutant SOD1 gene within astrocytes (Yamanaka et al., 2008), microglia (Beers et al., 2006; Boillee et al., 2006), or NG2 progenitors of the oligodendrocyte lineage (Kang et al., 2010) dramatically extends survival in the SOD1 mouse model of ALS. Cumulatively, these studies suggest that glial cells expressing mutant SOD1 play an active role in the death of motor neurons in this mouse model. Further direct evidence of glial involvement in ALS comes from in vitro studies showing astrocytes or microglia isolated from mutant SOD1 mice induce the death of healthy, wild-type motor neurons in culture (Di Giorgio et al., 2007; Nagai et al., 2007; Xiao et al., 2007). Lastly, evidence also exists to suggest glia generate a toxic environment for motor neurons in human ALS, even in sporadic cases. ALS patients undergo loss of the astrocytic glutamate transporter EAAT2 which may contribute to glutamate-mediated excitotoxic motor neuron death (Rothstein et al., 1992; Rothstein et al., 1995). Additionally, astrocytes derived from postmortem ALS spinal cord tissue cause the death of healthy, wild-type motor neurons in culture (Haidet-Phillips et al., 2011; Re et al., 2014). Taken together, these studies suggest that glial cells create a harmful microenvironment for motor neurons and thus, stem cell-derived motor neurons transplanted to the spinal cord of ALS patients may not have the chance to survive and reestablish connections with the muscle.

As an alternative to replacing dying motor neurons, stem cells are increasingly becoming recognized for their neuroprotective potential. Stem cells can differentiate not only into neurons, but also into glial cells which can function to support and protect vulnerable neuron populations. Likewise, stem cells can release trophic factors as well as modulate the inflammatory microenvironment to promote neuronal survival. For these reasons, we and others within the 
ALS field have focused on strategies to improve the microenvironment of the spinal cord with the goal of preserving the survival of remaining motor neurons and slowing the progression of disease.

\section{Development of stem cell therapy for ALS}

As our technical and biological understanding of stem cells continues to advance, there is an ever-growing number of stem cell types which can be exploited for cellular therapies. Since our goal for ALS is to modulate the glial environment for motor neuron protection, we have sought to use a progenitor stem cell type, which has the capacity to differentiate into glial lineages. Various groups have explored several progenitor types for stem cell transplantation. However, there is a strong rationale for transplantation of glial-restricted progenitor cells given that they can differentiate into astrocytes or oligodendrocytes (cell types which become dysfunctional during ALS). Glial-restricted progenitors are derived from neuroepithelial stem cells and can be isolated from the embryonic rodent or human CNS (Rao and Mayer-Proschel, 1997; Rao et al., 1998). Glial-restricted progenitors can be purified using the A2B5 cell surface antigen, expanded in vitro under GLP conditions, and can survive freeze-thaw cycles necessary for shipment of the cells to the transplantation site (Sandrock et al., 2010). Importantly, after transplantation, both rodent and human glial-restricted progenitors can survive, engraft, and differentiate into astrocytes and oligodendrocytes in the spinal cord of ALS rodent models (Lepore et al., 2008; Lepore et al., 2011). In the SOD1 ${ }^{\mathrm{G} 93 \mathrm{~A}}$ rat model of ALS, rodent glialrestricted progenitors attenuated motor neuron loss, slowed declines in forelimb motor and respiratory physiological function, and extended survival (Lepore et al., 2008). The SOD1 ${ }^{\text {G93A }}$ rats engrafted with glial-restricted progenitors also displayed reduced activation of immunemediating microglia. Neuroprotection was shown to be mediated in part by restoration of normal 
astrocytic glutamate transporter levels via transplanted glial-restricted progenitors, which had differentiated into astrocytes (Lepore et al., 2008). Transplantation of human glial-restricted progenitors into the $\mathrm{SOD} 1^{\mathrm{G} 93 \mathrm{~A}}$ mouse model was not able to extend survival or improve phenotypic measures of disease (Lepore et al., 2011). The lack of phenotypic improvement in that study may be due to several factors including the potential incompatibility of human transplant-derived beneficial factors with the mouse system, the rapidly progressive nature of the $\mathrm{SOD} 1^{\mathrm{G} 93 \mathrm{~A}}$ mouse model, and the extended timeframe for differentiation to human glia compared to the more rapid differentiation of mouse glial-restricted progenitors to astrocytes. Nevertheless, the human glial-restricted progenitors were able to survive and migrate diffusely throughout the gray and white matter and differentiate to astrocytes and cells of the oligodendrocyte lineage (Lepore et al., 2011). For these reasons as well as their safety and tolerability in toxicology studies in rodents and mini pigs (unpublished), glial-restricted progenitors are being pursued as a cellular therapy for ALS patients.

Similarly, other groups have explored different types of progenitor cells as stem cell sources for ALS therapy. Neural stem cells serve as the endogenous stem cell of the CNS which are multipotent and capable of differentiating to neurons, astrocytes, or oligodendrocytes. Neural stem cells can be obtained from fetal tissue or adult tissue by biopsy, giving multiple routes for generation of these stem cells. Neural stem cells derived from the human fetal spinal cord were transplanted to the spinal cord of the SOD $1^{\mathrm{G} 93 \mathrm{~A}}$ rat model where they differentiated into neurons, improved motor function, and extended survival (Xu et al., 2006). Although these transplanted neural stem cells differentiate mainly into neurons rather than glia (Yan et al., 2007), the newly generated neurons in this study did not replace the degenerated neuromuscular units. Instead, the proposed mechanism of action was derived to be through expression and release of protective 
growth factors from the transplanted stem cells and their neuronal progeny (Xu et al., 2006).

Subsequent studies showed an even greater therapeutic benefit when the human neural stem cells were targeted to not only the lumbar spinal cord, but also cervical segments as well (Xu et al., 2011). Additionally, experiments performed by Teng et al. showed that transplanted human neural stem cells could attenuate the disease course of SOD ${ }^{\mathrm{G} 93 \mathrm{~A}}$ mice including improved motor performance, respiratory function, pathological measures, and increased survival (Teng et al., 2012). The authors also performed a consortium-based meta-analysis of eleven independent studies which showed across-the-board prolonged survival and preservation of motor function in $\mathrm{SOD} 1^{\mathrm{G} 93 \mathrm{~A}}$ mice receiving neural stem cell transplants.

In addition to using neural stem cells for their capacity to differentiate into cell types of interest, other investigators are engineering these cells to secrete growth factors that may help to support neuron survival. Some of the pioneering work in this field has been performed by the Svendsen laboratory largely beginning with work in 2005 showing that human neural progenitor cells modified by lentiviral transduction to secrete glial-derived neurotrophic factor (GDNF) were able to integrate within the spinal cords of SOD $1^{\mathrm{G} 93 \mathrm{~A}}$ rats following intraspinal transplantation (Klein et al., 2005). This provided a platform for further studies by this group demonstrating the utility of these cells for providing motor neuron protection (Suzuki et al., 2007) as well as helping to maintain respiratory function in this model (Nichols et al., 2013). Additional studies utilizing human neural stem cells secreting vascular endothelial growth factor (VEGF) and transplanted intrathecally showed that human neural stem cells could migrate from the CSF space into the spinal cord parenchyma to provide neuroprotection (Hwang et al., 2009). This intrathecal approach could provide a more attractive alternative to direct intraspinal transplantation. Another study, however, using human neural progenitors engineered to express 
brain-derived neurotrophic factor (BDNF), insulin-like growth factor-1 (IGF-1), VEGF, neurotrophin-3 (NT-3), or GDNF, showed that while neuroprotection of motor neurons occurred in this ALS model, there was no concomitant maintenance of function (Park et al., 2009). That finding was similar to that of Suzuki and colleagues (Suzuki et al., 2008) who suggested that intramuscular delivery using GDNF mesenchymal stem cells in combination with spinal cord delivery of human neural stem cells expressing GDNF was required to see an improvement in SOD1 ${ }^{\mathrm{G} 93 \mathrm{~A}}$ rodent function. These studies suggest that growth factor secretion by neural stem cells is an attractive approach for motor neuron protection but that combined strategies of both central and peripheral delivery of growth factors may be a superior strategy.

In 2009, the FDA approved an open-label, first-in-human, feasibility and safety study for the transplantation of human spinal cord-derived neural stem cells into ALS patients. In total, 15 ALS patients received stem cell transplants with a risk-escalation scheme whereby the first patients were non-ambulatory ALS patients who received transplants to the lumbar spinal cord, followed by ambulatory patients receiving lumbar transplants, concluding with a cohort of patients receiving cervical transplants including some patients whom previously received transplants to their lumbar spinal cord (Feldman et al., 2014; Glass et al., 2012). This Phase I trial completed in 2013 demonstrated that the injection procedure and engrafted human neural stem cells were safe and well-tolerated by ALS patients who showed no acceleration of disease progression (Feldman et al., 2014; Glass et al., 2012). Phase II of this trial is currently underway and has paved the way for future stem cell trials using a similar intraparenchymal delivery scheme.

In addition to fetal-derived or adult-derived stem cell sources, many of the same stem cell types can be derived from human embryonic stem cells differentiated toward a neural lineage. 
Although human embryonic stem cell-derived oligodendrocyte progenitors have been transplanted in clinical trial for spinal cord injury patients, ethical issues remain with the use of these cells. As an alternative, pluripotent stem cells can now be created by reprogramming adult cell types to generate human induced pluripotent stem cells (Takahashi and Yamanaka, 2006; Takahashi et al., 2007). Human induced pluripotent stem cells are self-renewing, largely expandable, and can differentiate to endoderm, ectoderm, or mesoderm germ lineages. Indeed, recent reports show that human induced pluripotent stem cells differentiated to neural progenitor lineages can be engrafted to the spinal cord of the SOD1 mouse model of ALS with modest phenotypic improvement (Kondo et al., 2014; Nizzardo et al., 2014). In addition to the avoidance of ethical issues, human induced pluripotent stem cells offer another potential advantage for cell therapy since they can be reprogrammed directly from cells isolated from a patient biopsy. In this regard, they provide a source for autologous stem cell transplantation which could potentially avoid immune-mediated rejection of transplanted stem cells. However, autologous stem cell transplantation for ALS patients must be approached with caution. At least for the case of SOD1 mutations, strong evidence suggests that the presence of a SOD1 genetic mutation may cause glia to become dysfunctional (Di Giorgio et al., 2007; Haidet-Phillips et al., 2011; Nagai et al., 2007). Therefore, any glia derived from autologous transplanted stem cells expressing the same mutation may likewise become aberrant. Although genetic mutations have not been identified for the majority of sporadic ALS patients, there exists the possibility that these patients harbor unknown genetic alterations yet to be identified that could be present in autologous-derived stem cells. For the known ALS-linked genetic mutations, ex-vivo gene-correction strategies such as zinc-finger nucleases or transcription activator-like effector nucleases (TALENs) remain a possibility to allow for autologous stem cell transplantation. Indeed, these approaches have 
already been applied to ALS patient-derived human induced pluripotent stem cells being studied in vitro (Chen et al., 2014; Kiskinis et al., 2014; Wainger et al., 2014). However, until more is understood about the potential genetic causes of sporadic ALS, autologous stem cell transplantation for sporadic ALS should be approached with caution.

\section{Challenges in translation of stem cell therapies}

With the range of stem cells being developed and early clinical success gauged by safety and tolerability of stem cell injections, the future is bright for the potential of stem cell therapy for ALS. However, as the initial hurdle of safety is cleared, significant questions remain as to the best approaches to develop stem cell therapies which are not only safe, but also efficacious. Additional obstacles include tackling the quickly progressive nature of the disease, the involvement of the entire neural axis in the disease process, and detection strategies to follow transplanted cells and assess whether they are accomplishing their mechanistic goals.

In comparison to neurodegenerative diseases with regionally discrete cell death, ALS patients display involvement of the complete motor axis from corticospinal motor neuron cell death in the motor cortex to spinal motor neuron cell death along the entire spinal cord. One approach is to target stem cell transplants to critical regions such as cervical segments encompassing phrenic motor neurons crucial for respiratory function. Our laboratory has shown that in SOD $1^{\mathrm{G} 93 \mathrm{~A}}$ rats, targeting the cervical spinal cord with glial-restricted progenitors results in improved phrenic nerve compound muscle action potentials (CMAP), a functional electrophysiological assay of diaphragm function (Lepore et al., 2008). Improvement of diaphragmatic function could have a significant meaning for ALS patients since respiratory decline usually signals end-stage ALS. Nonetheless, quality of life is also of concern for patients 
and thus targeting of lumbar and cervical pools controlling limb muscles as well as bulbar motor neurons responsible for speaking and swallowing is also a weighty concern.

Intraparenchymal injection has been the method of choice for delivery of human neural stem cells to ALS patients in the first clinical trial for these cells in the US (Feldman et al., 2014; Glass et al., 2012). The drawback of this technique is that the distribution is limited by the cells' ability to migrate from the site of injection, requiring multiple injections to sufficiently target an area of the spinal cord. Intraparenchymal injection also poses potential safety risks pertaining to accidental disruption of the needle during injection. In order to provide a more stabilized platform for stereotaxic injection as well as improve accuracy to target the spinal cord ventral horn, a unique microinjection platform was designed using pigs as a model for the human spinal cord (Raore et al., 2011; Riley et al., 2009; Riley et al., 2011). After the device was deemed safe in pigs, it was used in the Phase I clinical trial sponsored by Neuralstem, Inc. to deliver human spinal cord-derived neural stem cells to the spinal cord of ALS patients by direct intraparenchymal injection (Feldman et al., 2014; Glass et al., 2012; Riley et al., 2012; Riley et al., 2014). Patients received up to 10 microinjections targeted to either the lumbar or cervical segments. Additionally, some patients underwent two surgeries with the first targeting the lumbar spinal cord and the second targeting the cervical spinal cord with these patients receiving up to 15 injections split between lumbar and cervical segments. Despite being invasive, the surgical procedure was well tolerated by ALS patients with no reported significant surgical complications and no acceleration of disease (Feldman et al., 2014; Glass et al., 2012; Riley et al., 2012; Riley et al., 2014). Thus, multiple, intraparenchymal, injections to deliver stem cells is safe at both lumbar and cervical levels. Phase II of this trial will focus on dose-escalation with ALS patients receiving up to a maximum of 40 injections to deliver $160 \times 10^{6}$ stem cells in total. 
The Neuralstem, Inc. trial has validated intraparenchymal delivery of neural stem cells to be a safe method to deliver stem cells to multiple regions of the spinal cord. However, not only do ALS patients suffer from spinal motor neuron loss, but corticospinal motor neurons in the motor cortex also degenerate throughout the disease process. The extent to which this upper motor neuron loss influences lower motor neuron health in ALS has not been widely investigated and motor cortex targeting for stem cell injections has been left out from the current clinical trial paradigms. Recent studies do suggest that protection of corticospinal motor neurons leads to enhanced survival of spinal motor neurons and neuromuscular junction preservation (Thomsen et al., 2014). In this study, the localized knockdown of mutant SOD1 in the motor cortex by viral vector delivery resulted in delayed disease onset, extended survival, and protection of lower spinal motor neurons in the SOD $1^{\mathrm{G} 93 \mathrm{~A}}$ rat model of ALS. Although the mechanisms for this lower motor neuron protection were not entirely elucidated, the potential mechanisms for the maintenance of these motor neuron pools deserves further study. Likewise, it will be important to know whether therapies directed to lower motor neurons similarly benefit the upper motor neuron pool. However, this study suggests that improved therapeutic success may be obtained by targeting stem cell engraftment to the motor cortex in addition to the spinal cord. Whether dual stem cell transplantation to the brain and spinal cord is feasible and safe in ALS patients will need to be closely examined.

Although parenchymal delivery of cells has been the standard, the development of minimally invasive strategies for the delivery of stem cells to the CNS is underway, To target the entire neural axis, the infusion of cells using an intra-arterial or intravenous approach has been considered; however, drawbacks include the potential for the cells to be absorbed by the peripheral organs before entering the CNS, the blood-brain-barrier preventing the cells from 
efficiently engrafting to the CNS, as well as the risk of a stem cell-induced stroke. Alternatively, intrathecal injection of stem cells could allow for more targeted administration of stem cells into the CNS although penetration of the cells from the cerebrospinal fluid into the parenchyma may still be a significant hurdle to overcome. In a recent study, Nizzardo et al. compared the delivery of human induced pluripotent stem cell-derived neural stem cells by either intrathecal or intravenous delivery in the SOD1 ${ }^{\mathrm{G} 93 \mathrm{~A}}$ mouse model of ALS. To enhance the homing potential of stem cells to enter the parenchyma after intravenous delivery, the authors used fluorescence activated cell sorting (FACS) to isolate a population of human induced pluripotent stem cellderived neural stem cells expressing the VLA4 integrin allowing for enhanced binding to the blood-brain-barrier via adhesion molecules (Nizzardo et al., 2014). Indeed, there was an increased therapeutic effect when the transplanted cells were administered by intravenous injection compared to intrathecal injection. These strategies are promising and would allow for increased targeting of the entire neural axis through a minimally invasive procedure.

Although secondary to safety concerns, another hurdle to overcome is detection and tracking of transplanted stem cells in patients. In animal studies, transplanted cells are typically labeled with a fluorescent reporter or detected immunohistochemically using human-specific antibodies to detect cells of human origin within rodent tissue. However, fluorochromes do not penetrate well through deep tissues, making in vivo imaging in humans difficult. Additionally, there are valid concerns related to introducing an artificial reporter system into stem cells for subsequent transplantation into humans.

In the Neuralstem, Inc. trial where human spinal cord neural stem cells were injected intraparenchymally to ALS patients, the cells were not labeled or tracked in vivo. The lack of an ability to track transplanted cells presents problems in critically evaluating whether the immune 
suppression regimen was sufficient to allow for graft survival. Additionally, the proposed mechanism of action for the transplanted cells based on rodent studies involved long-term engraftment to the spinal cord, differentiation to neurons, and secretion of supportive growth factors. It is difficult to evaluate whether the transplanted cells accomplished these goals without a method to track or label the cells. To address this problem, Tadesse et al. analyzed the postmortem spinal cords of patients in the Neuralstem, Inc. trial and used fluorescence in situ hybridization (FISH) to identify transplanted cells which were of male origin (XY) in the female patient (XX) who received the stem cell transplant (Tadesse et al., 2014). In addition to using FISH to localize transplanted cells in the one female patient included in the trial, the authors successfully used quantitative real-time PCR to detect donor stem cell-derived DNA in the spinal cord of all six deceased patients surviving from 6 months up to 2.5 years from the time of transplantation. These results are encouraging and suggest the transplanted human spinal cordderived neural stem cells can survive long-term in the spinal cord of ALS patients, most of whom even discontinued their immune-suppressive regimen at various times post-transplantation (Tadesse et al., 2014).

Due to the high spatial resolution, there are currently methods in development to use magnetic resonance imaging (MRI) in order to track transplanted cells in vivo. For these studies, cells can be labeled in vitro using nanoparticles to load cells with superparamagnetic iron oxide which increases the intracellular iron concentration allowing for detection by MRI (Bulte and Kraitchman, 2004). This technique has been used to track mesenchymal stem cells transplanted by intrathecal injection to the spinal cord of ALS patients (Karussis et al., 2010). Although promising, MRI-based cell tracking is still in its infancy and further work will be needed to determine the sensitivity, safety, and the long-term ability for tracers to stay confined to 
transplanted cells versus being transferred to immune or other phagocytic cells (Ahrens and Bulte, 2013).

Lastly, as discussed previously, studies have shown that glial cells in the ALS spinal cord create a toxic environment for motor neurons; hence, motor neuron replacement strategies may be plagued by the healthy, transplant-derived motor neurons succumbing to the damaging environment. Similarly, engrafted stem cells or stem cell-derived glia could be subject to the same toxic milieu after transplantation. How engrafted stem cells or stem cell-derived glia react to the neurodegenerative environment of the ALS spinal cord has been largely unexplored. To begin to address this question, we transplanted human glial-restricted progenitors to the spinal cords of wild-type and SOD $1^{\mathrm{G} 93 \mathrm{~A}}$ mice and asked how the ALS spinal cord affects engraftment, differentiation, and gene expression of the human glial-restricted progenitors. Our studies showed that human glial-restricted progenitors survived, migrated, and differentiated to astrocytes in the SOD $1^{\mathrm{G} 93 \mathrm{~A}}$ spinal cord similarly to how human glial-restricted progenitors engrafted to the wild-type spinal cord (Haidet-Phillips et al., 2014). Using human-specific probes designed using NanoString® technology, we assessed the in vivo gene profile of human glialrestricted progenitors at different time points after transplantation to the wild-type versus SOD1 ${ }^{\mathrm{G} 93 \mathrm{~A}}$ spinal cord. No significant differences were found in the expression of astrocyte, oligodendrocyte, or progenitor genes by the human glial-restricted progenitors engrafted to the SOD $1^{\mathrm{G} 93 \mathrm{~A}}$ spinal cord versus gene expression by human glial-restricted progenitors in the wildtype spinal cord (Haidet-Phillips et al., 2014). The engrafted astrocytes also did not display characteristics of mutant SOD1-expressing astrocytes such as GFAP upregulation and glutamate transporter loss. In fact, a modest upregulation of the EAAT2 glutamate transporter was observed in the transplanted cells. Overall, these studies suggest that human glial-restricted 
progenitors are capable of retaining their cell-autonomous phenotype even when transplanted into a neurodegenerative environment which is encouraging considering these cells are in preclinical development for ALS patients.

\section{$\underline{\text { Conclusions }}$}

While many challenges still remain, incredible progress on the clinical forefront has been accomplished in recent years, highlighted by the recent completion of the first-in-human, Phase I clinical trial for transplantation of neural stem cells to the spinal cord of ALS patients (Feldman et al., 2014; Glass et al., 2012). This clinical trial proved that intraspinal injection of human neural stem cells is safe and feasible for ALS patients, paving the way for future stem cell therapies by creating a path through regulatory obstacles. It still remains to be seen whether stem cell transplantation will positively affect the disease course in ALS patients. However, preclinical studies suggest that stem cells can significantly impact ALS including our own studies using human glial-restricted progenitors which have recently received IND approval (Lepore et al., 2008; Xu et al., 2006; Xu et al., 2011). Additionally, our knowledge of the disease has advanced significantly with the recent identification of new genetic factors such as the C9ORF72 expansion and the development of human induced pluripotent stem cell technology (DeJesus-Hernandez et al., 2011; Renton et al., 2011; Richard and Maragakis, 2014; Takahashi and Yamanaka, 2006; Takahashi et al., 2007). These recent advancements may lead to the development of better animal models or humanized models for the study of disease mechanisms. Improved preclinical, proof-of-concept studies using better models and more rigorous controls should assist in helping to choose the most promising stem cell therapies to test in the clinic. In addition to improved preclinical studies, the development of methods to track and identify stem cells engrafted to human patients will allow for more precise clinical assessment to determine 
whether stem cells are meeting their mechanistic goals in humans as predicted from rodent studies. Overall, initial studies in humans have shown that the opportunity exists for stem cell transplantation to be of true benefit for ALS patients. The future will determine the best cellular types and paradigms for transplantation and tracking, and ultimately, whether stem cells can promote motor neuron survival for patients.

Acknowledgements: NJM is supported by NIH/NIHDS (U01NS062713), Department of Defense ALS Research Program, The Robert Packard Center for ALS Research, and The Maryland Stem Cell Research Fund. AHP is supported by the Milton Safenowitz Post-doctoral Fellowship from the ALS Association and a Career Development Award from the Muscular Dystrophy Association.

N.J. Maragakis is an unpaid consultant to Q Therapeutics, Inc. and is the Principal Investigator on a NIH/NINDS U01 with Q Therapeutics, Inc.

\section{$\underline{\text { References }}$}

Ahrens, E.T., Bulte, J.W., 2013. Tracking immune cells in vivo using magnetic resonance imaging. Nat Rev Immunol. 13, 755-63.

Beers, D.R., et al., 2006. Wild-type microglia extend survival in PU.1 knockout mice with familial amyotrophic lateral sclerosis. Proc Natl Acad Sci U S A. 103, 16021-6.

Bensimon G, L.L., Meininger V, ALS, Group RS., 1994. A controlled trial of Riluzole in amyotrophic lateral sclerosis. New England Journal of Medicine. 330, 585-591.

Boillee, S., et al., 2006. Onset and Progression in Inherited ALS Determined by Motor Neurons and Microglia. Science. 312, 1389-92.

Bulte, J.W., Kraitchman, D.L., 2004. Iron oxide MR contrast agents for molecular and cellular imaging. NMR Biomed. 17, 484-99.

Chen, H., et al., 2014. Modeling ALS with iPSCs reveals that mutant SOD1 misregulates neurofilament balance in motor neurons. Cell Stem Cell. 14, 796-809.

Corti, S., et al., 2007. Neural stem cells LewisX+ CXCR4+ modify disease progression in an amyotrophic lateral sclerosis model. Brain. 130, 1289-305. 
DeJesus-Hernandez, M., et al., 2011. Expanded GGGGCC hexanucleotide repeat in noncoding region of C9ORF72 causes chromosome 9p-linked FTD and ALS. Neuron. 72, 245-56.

Di Giorgio, F.P., et al., 2007. Non-cell autonomous effect of glia on motor neurons in an embryonic stem cell-based ALS model. Nat Neurosci. 10, 608-14.

Feldman, E.L., et al., 2014. Intraspinal neural stem cell transplantation in amyotrophic lateral sclerosis: phase 1 trial outcomes. Ann Neurol. 75, 363-73.

Glass, J.D., et al., 2012. Lumbar intraspinal injection of neural stem cells in patients with amyotrophic lateral sclerosis: results of a phase I trial in 12 patients. Stem Cells. 30, 1144-51.

Gurney, M.E., et al., 1994. Motor neuron degeneration in mice that express a human $\mathrm{Cu}, \mathrm{Zn}$ superoxide dismutase mutation. Science. 264, 1772-5.

Haidet-Phillips, A.M., et al., 2011. Astrocytes from familial and sporadic ALS patients are toxic to motor neurons. Nat Biotechnol. 29, 824-8.

Haidet-Phillips, A.M., et al., 2014. Human glial progenitor engraftment and gene expression is independent of the ALS environment. Exp Neurol. 264C, 188-199.

Hwang, D.H., et al., 2009. Intrathecal transplantation of human neural stem cells overexpressing VEGF provide behavioral improvement, disease onset delay and survival extension in transgenic ALS mice. Gene Ther. 16, 1234-44.

Jaarsma, D., et al., 2008. Neuron-specific expression of mutant superoxide dismutase is sufficient to induce amyotrophic lateral sclerosis in transgenic mice. J Neurosci. 28, 2075-88.

Kang, S.H., et al., 2010. NG2+ CNS glial progenitors remain committed to the oligodendrocyte lineage in postnatal life and following neurodegeneration. Neuron. 68, 668-81.

Karussis, D., et al., 2010. Safety and immunological effects of mesenchymal stem cell transplantation in patients with multiple sclerosis and amyotrophic lateral sclerosis. Arch Neurol. 67, 1187-94.

Kiskinis, E., et al., 2014. Pathways disrupted in human ALS motor neurons identified through genetic correction of mutant SOD1. Cell Stem Cell. 14, 781-95.

Klein, S.M., et al., 2005. GDNF delivery using human neural progenitor cells in a rat model of ALS. Hum Gene Ther. 16, 509-21.

Kondo, T., et al., 2014. Focal transplantation of human iPSC-derived glial-rich neural progenitors improves lifespan of ALS mice. Stem Cell Reports. 3, 242-9.

Lepore, A.C., et al., 2008. Focal transplantation-based astrocyte replacement is neuroprotective in a model of motor neuron disease. Nat Neurosci. 11, 1294-301.

Lepore, A.C., et al., 2011. Human glial-restricted progenitor transplantation into cervical spinal cord of the SOD1 mouse model of ALS. PLoS One. 6, e25968.

Lino, M.M., Schneider, C., Caroni, P., 2002. Accumulation of SOD1 mutants in postnatal motoneurons does not cause motoneuron pathology or motoneuron disease. J Neurosci. 22, 4825-32.

Lopez-Gonzalez, R., Kunckles, P., Velasco, I., 2009. Transient recovery in a rat model of familial amyotrophic lateral sclerosis after transplantation of motor neurons derived from mouse embryonic stem cells. Cell Transplant. 18, 1171-81.

Martin, L.J., Liu, Z., 2007. Adult olfactory bulb neural precursor cell grafts provide temporary protection from motor neuron degeneration, improve motor function, and extend survival in amyotrophic lateral sclerosis mice. J Neuropathol Exp Neurol. 66, 1002-18. 
Nagai, M., et al., 2007. Astrocytes expressing ALS-linked mutated SOD1 release factors selectively toxic to motor neurons. Nat Neurosci. 10, 615-622.

Nichols, N.L., et al., 2013. Intermittent hypoxia and stem cell implants preserve breathing capacity in a rodent model of amyotrophic lateral sclerosis. Am J Respir Crit Care Med. $187,535-42$.

Nizzardo, M., et al., 2010. Human motor neuron generation from embryonic stem cells and induced pluripotent stem cells. Cell Mol Life Sci. 67, 3837-47.

Nizzardo, M., et al., 2014. Minimally invasive transplantation of iPSC-derived ALDHhiSSCloVLA4+ neural stem cells effectively improves the phenotype of an amyotrophic lateral sclerosis model. Hum Mol Genet. 23, 342-54.

Park, S., et al., 2009. Growth factor-expressing human neural progenitor cell grafts protect motor neurons but do not ameliorate motor performance and survival in ALS mice. Exp Mol Med. 41, 487-500.

Peljto, M., Wichterle, H., 2011. Programming embryonic stem cells to neuronal subtypes. Curr Opin Neurobiol. 21, 43-51.

Pramatarova, A., et al., 2001. Neuron-specific expression of mutant superoxide dismutase 1 in transgenic mice does not lead to motor impairment. J Neurosci. 21, 3369-74.

Rao, M.S., Mayer-Proschel, M., 1997. Glial-restricted precursors are derived from multipotent neuroepithelial stem cells. Dev Biol. 188, 48-63.

Rao, M.S., Noble, M., Mayer-Proschel, M., 1998. A tripotential glial precursor cell is present in the developing spinal cord. Proc Natl Acad Sci U S A. 95, 3996-4001.

Raore, B., et al., 2011. Cervical multilevel intraspinal stem cell therapy: assessment of surgical risks in Gottingen minipigs. Spine (Phila Pa 1976). 36, E164-71.

Re, D.B., et al., 2014. Necroptosis Drives Motor Neuron Death in Models of Both Sporadic and Familial ALS. Neuron.

Renton, A.E., et al., 2011. A hexanucleotide repeat expansion in C9ORF72 is the cause of chromosome 9p21-linked ALS-FTD. Neuron. 72, 257-68.

Richard, J.P., Maragakis, N.J., 2014. Induced pluripotent stem cells from ALS patients for disease modeling. Brain Res.

Riley, J., et al., 2009. Cervical spinal cord therapeutics delivery: preclinical safety validation of a stabilized microinjection platform. Neurosurgery. 65, 754-61; discussion 761-2.

Riley, J., et al., 2012. Intraspinal stem cell transplantation in amyotrophic lateral sclerosis: a phase I safety trial, technical note, and lumbar safety outcomes. Neurosurgery. 71, 40516; discussion 416.

Riley, J., et al., 2014. Intraspinal stem cell transplantation in amyotrophic lateral sclerosis: a phase I trial, cervical microinjection, and final surgical safety outcomes. Neurosurgery. 74, 77-87.

Riley, J.P., et al., 2011. Platform and cannula design improvements for spinal cord therapeutics delivery. Neurosurgery. 69, ons 147-54; discussion ons 155.

Rothstein, J.D., Martin, L.J., Kuncl, R.W., 1992. Decreased glutamate transport by the brain and spinal cord in amyotrophic lateral sclerosis. N Engl J Med. 326, 1464-8.

Rothstein, J.D., et al., 1995. Selective loss of glial glutamate transporter GLT-1 in amyotrophic lateral sclerosis. Ann Neurol. 38, 73-84.

Sandrock, R.W., et al., 2010. Isolation, characterization and preclinical development of human glial-restricted progenitor cells for treatment of neurological disorders. Regen Med. 5, 381-94. 
Suzuki, M., et al., 2007. GDNF secreting human neural progenitor cells protect dying motor neurons, but not their projection to muscle, in a rat model of familial ALS. PLoS One. 2, e689.

Suzuki, M., et al., 2008. Direct muscle delivery of GDNF with human mesenchymal stem cells improves motor neuron survival and function in a rat model of familial ALS. Mol.Ther. 16, 2002-2010.

Tadesse, T., et al., 2014. Analysis of graft survival in a trial of stem cell transplant in ALS. Ann Clin Transl Neurol. 1, 900-8.

Takahashi, K., Yamanaka, S., 2006. Induction of pluripotent stem cells from mouse embryonic and adult fibroblast cultures by defined factors. Cell. 126, 663-676.

Takahashi, K., et al., 2007. Induction of pluripotent stem cells from adult human fibroblasts by defined factors. Cell. 131, 861-872.

Teng, Y.D., et al., 2012. Multimodal actions of neural stem cells in a mouse model of ALS: a meta-analysis. Sci Transl Med. 4, 165 ra164.

Thomsen, G.M., et al., 2014. Delayed Disease Onset and Extended Survival in the SOD1G93A Rat Model of Amyotrophic Lateral Sclerosis after Suppression of Mutant SOD1 in the Motor Cortex. J Neurosci. 34, 15587-600.

Wainger, B.J., et al., 2014. Intrinsic membrane hyperexcitability of amyotrophic lateral sclerosis patient-derived motor neurons. Cell Rep. 7, 1-11.

Xiao, Q., et al., 2007. Mutant SOD1(G93A) microglia are more neurotoxic relative to wild-type microglia. J Neurochem. 102, 2008-19.

Xu, L., et al., 2006. Human neural stem cell grafts ameliorate motor neuron disease in SOD-1 transgenic rats. Transplantation. 82, 865-875.

Xu, L., et al., 2011. Dual transplantation of human neural stem cells into cervical and lumbar cord ameliorates motor neuron disease in SOD1 transgenic rats. Neurosci Lett. 494, 2226.

Yamanaka, K., et al., 2008. Astrocytes as determinants of disease progression in inherited amyotrophic lateral sclerosis. Nat Neurosci. 11, 251-3.

Yan, J., et al., 2007. Extensive neuronal differentiation of human neural stem cell grafts in adult rat spinal cord. PLoS Med. 4, e39. 